\title{
Measuring the Maximum Power of an ex vivo Engineered Muscle Tissue With Isovelocity Shortening Technique
}

\author{
Simona Pisu, Marianna Cosentino, Ludovica Apa, Antonio Musarò, Emanuele Rizzuto ${ }^{\circledR}$, and Zaccaria Del Prete
}

\begin{abstract}
The final aim of muscle tissue engineering (TE) is to create a new tissue able to restore the functionality of impaired muscles once transplanted in the site of injury. Therefore, functional contractile properties close to that of healthy muscles are desirable to allow for a good compatibility and a proper functional contribution. Since skeletal muscles deal with locomotion during their normal activity, an accurate measurement of ex vivo muscle engineered tissues' isotonic properties is crucial. In this paper, we devised an experimental system to measure the mechanical power generated by an ex vivo muscle engineered tissue, the X-MET, based on the isovelocity contraction technique. The X-MET is developed without the use of any scaffolds, so that its mechanical properties are not affected by endogenous components. Our experiments allowed for delimiting the ranges of shortening and shortening velocity for which the tissue is able to generate and maintain power for the entire stimulation, which is the condition that better reproduces muscle physiological activity. Then, we measured the power generated by the X-MET and fit the experimental results to the Hill's equation usually employed for modeling the force-velocity relationship of skeletal muscles. The use of this model yielded to the measurement of maximum power and maximum shortening velocity. Results revealed that most of the isotonic properties were consistent with that proposed in the literature for slow-twitch muscles; in particular, the $\mathrm{X}$-METs were able to generate a maximum power of $2.08 \pm 0.78 \mathrm{~W} / \mathrm{kg}$ and had a maximum shortening velocity of $1.84 \pm 0.57 \mathrm{~L}_{0} / \mathrm{s}$, on average.
\end{abstract}

Index Terms-Hill's model, isovelocity test, power measurement, skeletal muscle, tissue engineering (TE).

\section{INTRODUCTION}

$\mathbf{R}$ EGENERATIVE medicine is an emerging field of research and therapy whose aim is to restore the impaired function of defective tissues and organs using stem cells through two different approaches: cell therapy and tissue engineering (TE). In particular, the aim of TE is to obtain a construct with structure and functionality close to that of the natural tissue to be replaced, to serve as a therapeutic

Manuscript received September 29, 2018; revised December 20, 2018; accepted February 20, 2019. The Associate Editor coordinating the review process was Domenico Grimaldi. (Corresponding author: Emanuele Rizzuto.)

S. Pisu, L. Apa, E. Rizzuto, and Z. Del Prete are with the Department of Mechanical and Aerospace Engineering, Sapienza University of Rome, 00184 Rome, Italy (e-mail: emanuele.rizzuto@uniroma1.it).

M. Cosentino and A. Musarò are with the Institute Pasteur-Cenci Bolognetti, DAHFMO-Unit of Histology and Medical Embryology, IIM, Sapienza University of Rome, 00161 Rome, Italy.

Color versions of one or more of the figures in this paper are available online at http://ieeexplore.ieee.org.

Digital Object Identifier 10.1109/TIM.2019.2904071 device. To do this, TE requires the interaction among three main components: cells, a matrix that supports cell growth, and all the mechanical and molecular signals that recapitulate the in vivo niche in which cells reside. Up to twenty years ago TE was limited only to the reconstruction of grafts of epithelia to treat skin and mucous membrane lesions. Subsequently, TE started approaching the generation of substitutes for repairing damaged tissues of the musculoskeletal system as bone, cartilage, and muscle [1], [2].

Skeletal muscle, as well as myocardium, is a complex tissue, in which the regenerative properties depend on the proliferative and differentiative potential of satellite stem cells. Senescence, denervation, removal of tumor tissue, injury, and dystrophy are examples of conditions that could make the regeneration processes insufficient for the complete recovery of skeletal muscle [3]. In this context, engineered tissues gained more and more relevance because of their potential to be used for injured and infarcted patients. Two different approaches are mainly employed to engineer skeletal and cardiac muscle: in vivo and in vitro [4], [5]. In the in vivo approach, myogenic or progenitor cells are seeded on a 3-D matrix, and the entire construct is then transplanted in order to allow for the differentiation of the progenitor cells into myotubes directly within the host tissue. In the second approach, cells are typically differentiated, in vitro, on a 3-D scaffold to generate an artificial construct to be reimplanted. An ideal 3-D scaffold for TE has high porosity, biocompatibility, and biodegradability [6]-[9]: to date, the most used scaffolds are synthetic biodegradable [10]-[12] and those directly based on the extra cellular matrix [13]-[15]. The use of spheroids [16], [17] as a 3-D in vitro model without the use of scaffold is also spreading, while the first 3-D muscle tissue realized without the use of an external support was proposed by Strohman et al. [18] and colleagues in 1990 [18]. Subsequently, Dennis et al. [19] developed a 3-D skeletal muscle tissue engineered with artificial tendons and, more recently, we proposed a 3-D engineered skeletal muscle obtained from mouse skeletal muscle primary culture without the use of any exogenous component, the X-MET [20].

The X-MET, ex vivo muscle engineered tissue, is a musclelike structure with a heterogeneous cell population, mainly composed of myoblasts, fibroblasts, nervous, and endothelial cells. In particular, fibroblasts are responsible of both the formation and maintenance of the 3-D organization and the 
satellite-like cells are surrounded by the basal lamina. This latter aspect is very interesting, as it indicates that, in the $\mathrm{X}$ MET structure, the satellite cell niche can be reconstructed in vitro. The resulting X-MET mimicked the structure and function of muscles more closely than classical 2-D approaches. In previous papers [20], [21], we measured several parameters characterizing the X-MET's isometric contractile capability, as the spontaneous contraction, the kinetic properties, and the force-frequency relationship. However, during normal activity skeletal muscle allows for locomotion of the body encountering isotonic contractions, so that the parameter that better describes muscle and muscle-like tissues functionality is the mechanical power [22], and new methods are continuously proposed to improve its measurements [23], [24]. The measurement of the generated power is, therefore, crucial, and can help improving the tissue formation to obtain the desired contractile response before transplanting. To the best of the authors' knowledge, no measurements of mechanical power has been performed for ex vivo muscle engineered tissues so far.

Within this context, the aim of the proposed work was to measure, for the first time, the maximum power generated by an $e x$ vivo engineered tissue, using the isovelocity shortening contraction as a testing technique. During isovelocity tests, the tissue is shortened at a fixed shortening velocity and electrically stimulated at the same time; the mechanical power is computed as the product of the maximum force generated by the tissue and the shortening velocity. This technique has been previously employed to measure fast and slow skeletal muscle power output [25]-[27], and we referred to the values of shortening and shortening velocity employed for slow-twitch muscles as initial values for our tests. After that, a series of tests have been performed to find out the optimal combinations of these parameters that allowed the X-MET to develop and maintain the generated force for the entire stimulation, which is the condition that better mimics the behavior of muscles during normal activity. The isovelocity shortening technique can be, therefore, employed to measure the power generated by any kind of muscle-like tissue, but the optimal testing parameters specific for the specimen to be tested have to be found out in advance.

A second aim of this paper was to obtain a modeling of the relationship between the force generated by the tissue and the shortening velocity, so to determine other crucial parameters of ex vivo muscle engineered tissues' contractility, as the maximum shortening velocity, the maximum power, and the values of force and velocity at which the tissue develops the maximum power. To do this, we applied the hyperbolic model proposed by Hill [28] in 1938 and currently employed to model the F-v relationship for skeletal and cardiac muscles.

\section{MATERIALS AND METHODS}

\section{A. Experimental System}

All experiments were conducted in accordance with the Italian Law, which governs the use of animals in experimentation, and the procedure was approved by the local

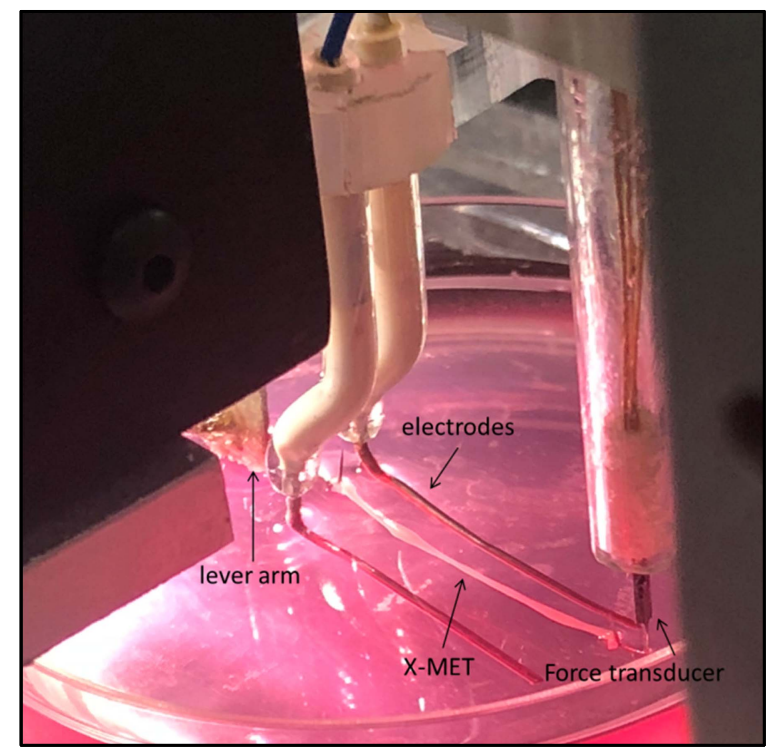

Fig. 1. Experimental setup employed to measure the X-MET's mechanical power.

ethics review board. The X-MET specimens were obtained from skeletal muscle primary cultures of C57BL/6 mice (4-5 weeks of age) following a protocol similar to that illustrated by Carosio et al. [20]. Briefly, a heterogeneous cell population was isolated from skeletal muscles of hind limbs with an apparatus for mechanical and enzymatic digestion (gentleMACS Octo Dissociator, MiltenyiBiotec). After seven days of culture in a collagen coated petri dish, the cells resulted in a monolayer that delaminates spontaneously. Once delaminated, the monolayer was pinned on a silicone coated dish using two 0.20 -mm-diameter stainless steel pins to induce a mechanical tension. After 2-3 days, the monolayer selfassembled into a 3-D-cylindrical structure and began to contract spontaneously. About 20 days after cell isolation, the specimen to be tested was mounted between the lever arm of an actuator/transducer (Aurora Scientific Inc. 300C) for length control, and a microforce transducer (Kronex AE801). For the entire duration of the experiment, the tissue was kept soaked in a differentiation medium and maintained at the temperature of $37^{\circ} \mathrm{C} \pm 0.3{ }^{\circ} \mathrm{C}$ with the use of a temperature control plate (Okolabs.r.l., H401). Two custom built platinum electrodes were located parallel to the specimen allowing the pulse stimulator (Aurora Scientific Inc. 701B) to elicit the tissue contraction inside the physiological solution. Fig. 1 shows a photograph of the experimental setup.

A software developed in LabVIEW 2012 allowed setting the test parameters, namely, the frequency and duration of the electrical stimulation, the shortening and the shortening velocity. Particular care was given to the synchronization between the electrical stimulation and the shortening: for all the tests performed we measured a delay lower than $1.2 \mathrm{~ms}$. Since the duration of the optimal electrical stimulations and shortenings have been measured to be in the range of $100-600 \mathrm{~ms}$, as shown below, this delay was considered negligible. 


\section{B. Experimental Testing Protocol}

The testing protocol has been carried out in four stages, as reported in [29]: 1) determination of the specimen optimal length; 2) measurement of tissue maximum force; 3) isovelocity tests; and 4) measurement of tissue maximum force for double-check of tissue fatigability. At the end of the protocol, the tissue was gently dried and its mass was measured with a Pioneer precision scale (Ohaus, Parsippany, NJ, USA) with an accuracy of $0.1 \mathrm{mg}$.

To determine the optimal length $\left(\mathrm{L}_{0}\right)$, the tissue was stimulated to generate the maximum twitch force with a series of single pulses ( $400 \mathrm{~mA}, 1.2 \mathrm{~ms}$ ), while changing its length. The $\mathrm{L}_{0}$ was measured with an analog caliper with an accuracy of $0.05 \mathrm{~mm}$, and was used for normalization of the imposed shortening, now expressed as $L / \mathrm{L}_{0} \%$, and of the shortening velocity, now expressed as $\mathrm{L}_{0} / \mathrm{s}$. The maximum isometric force was measured stimulating the tissue with a $0.7 \mathrm{~s}$ train of $1 \mathrm{~ms}$ pulses delivered at $80 \mathrm{~Hz}$ [30].

To measure the maximum power, the tissue was subjected to a series of isovelocity shortening tests: the specimen was electrically stimulated, while shortened at a constant speed; at the same time the force generated during the shortening was measured. At the end of the stimulation (and of the shortening), the tissue was held isometrically, allowing for relaxing before returning to its resting length [26]. When performing isovelocity shortening tests, the crucial parameters to be set are the shortening and shortening velocity which, in turn, determine the duration of the electrical stimulation: the tissue is able to develop and maintain the generated force for the entire duration of the stimulation only for particular combinations of these parameters [25]. Therefore, our protocol involved several subsequent stimulations for different combinations of shortening and shortening velocity. Since a similar protocol has never been applied on ex vivo skeletal muscle engineered tissues, we needed to find out the range of stimulation parameters able to elicit the requested contractile response. All the X-MET's mechanical properties previously measured were close to Soleus muscle and red fiber bundles [20], so that we took the values of shortening and shortening velocity employed in the literature for these muscle types as initial reference values. In particular, shortenings around $10 \%$ and shortening velocity around $1 \mathrm{~L}_{0} / \mathrm{s}$ [25]-[27] are the most used for slow-twitch muscle fiber tissues.

In view of this, we subjected one X-MET to several combinations of shortenings and shortening velocities in the range of $1 \% \mathrm{~L}_{0}-20 \% \mathrm{~L}_{0}$, and in the range of $0.1 \mathrm{~L}_{0} / \mathrm{s}-2 \mathrm{~L}_{0} / \mathrm{s}$ respectively, to analyze the force response of the tissue. The power developed by the tissue was then computed with a program written in MATLAB as the maximum force developed during each stimulation multiplied by the shortening velocity.

Once the maximum and minimum values of shortening and shortening velocity for which the tissue was able to develop and maintain the force for the entire duration of the stimulation were determined, we applied a systematic testing protocol on $11 \mathrm{X}$-MET samples to measure the maximum power. The shortenings and shortening velocities employed were combinations of the following values: $5 \%, 7 \%, 10 \%$, and $12 \%$ of $\mathrm{L}_{0}$, and $0.25,0.5,0.75$, and $1 \mathrm{~L}_{0} / \mathrm{s}$. Of note, the difference between the first and the last tetanic stimulation resulted always lower than $10 \%$, highlighting that the tissue did not undergo to fatigue during these tests.

\section{Modeling the Force-Velocity Relationship}

The relationship between the shortening velocity and the force is crucial to evaluate the contractility of a musclelike construct. This relationship allows for an estimation of the maximum shortening velocity of the tissue, which is the velocity that occurs in a condition of shortening against a null load. It also yields to an estimation of the maximum power, and the optimal force and shortening velocity, namely, the conditions for which the maximum power is generated [28]. To do this, we used the Hill's model widely employed for skeletal muscles, which hypothesizes a hyperbolic relationship between force and velocity. This model has been shown to provide a valid prediction of most of the parameters associated with muscle contractility, including those obtained from isotonic shortening contractions [31]. We developed a MATLAB script to fit the experimental points to the following:

$$
F(v)=\frac{-a *(v+b)+c}{(v+b)}
$$

where $F(v)$ is the maximum force measured during each shortening normalized by the maximum isometric force, and $v$ is the shortening velocity imposed during each contraction. Once the model was obtained, the maximum shortening velocity was determined for $F=0$. The power was computed by multiplying the force values resulting from the model by the shortening velocities, in the range of 0 and $v_{\max }$. The optimal force and shortening velocity were computed as the values for which the maximum power was obtained. The power was then normalized by the tissue weight to allow for a proper comparison with mouse skeletal muscles.

\section{Statistical analysis}

Differences in the values of normalized power were evaluated with repeated measures two-way ANOVA using the factors shortening and shortening velocity. Indeed, in our experimental protocol, each specimen has been tested for four different shortenings and four different shortening velocities. When the two-way ANOVA indicated significant differences, we used multiple Fisher test to look for statistical differences. Statistical analysis was performed with GraphPad Prism 6.0 and differences were considered significant when $p<0.05$. Data reported all through the manuscript are expressed as mean $\pm \mathrm{SD}$.

\section{RESULTS}

\section{A. Identification of Stimulation Parameters}

The power generated by a skeletal muscle-like tissue during isovelocity tests is strictly dependent on the amount of shortening the tissue is allowed to achieve and the shortening velocity. Only a proper combination of these two parameters yields the tissue to develop the maximum power and keep it for the entire 


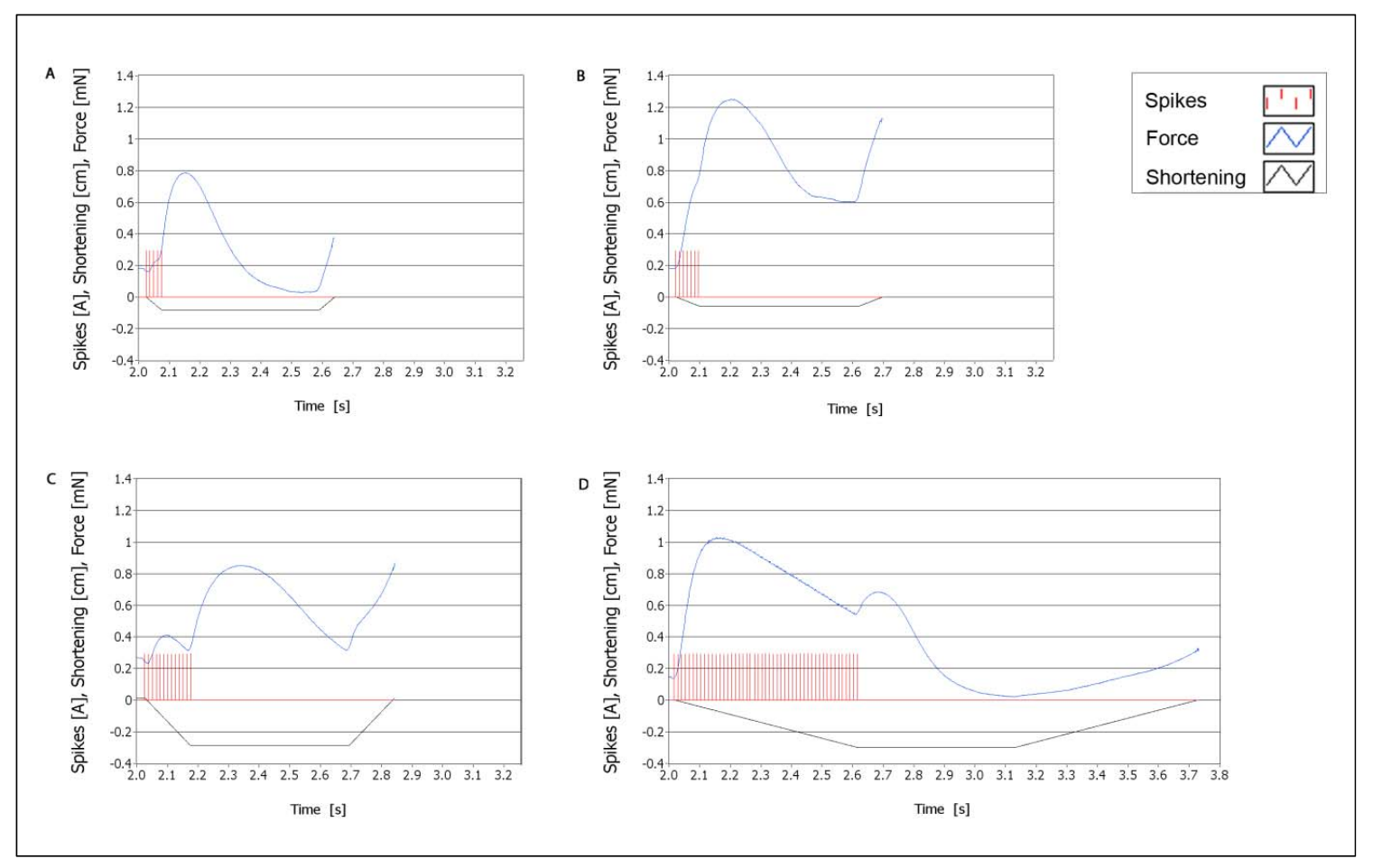

Fig. 2. Examples of the four stimulation conditions outside the optimal ranges of shortening and shortening velocity. (a) Small shortening and high shortening velocity $\left(3 \%\right.$ and $1 \mathrm{~L}_{0} / \mathrm{s}$ ). (b) Small shortening and low shortening velocity (3\% and $0.2 \mathrm{~L} 0 / \mathrm{s}$ ). (c) Big shortening and high shortening velocity (15\% and $\left.1.2 \mathrm{~L}_{0} / \mathrm{s}\right)$. (d) Big shortening and low shortening velocity $\left(15 \%\right.$ and $\left.0.2 \mathrm{~L}_{0} / \mathrm{s}\right)$.

duration of the stimulation. Fig. 2 shows four combinations of these parameters for which the force generated by the X-MET is not suitable for power measurement.

First, our results showed that a small amount of shortening did not allow for a sufficient stimulation time, both for high [Fig. 2(a)] and low [Fig. 2(b)] shortening velocity. In the first case, the stimulation time is excessively short, and the tissue does not generate a significant force at all during the electrical stimulation (that is also the shortening period). In the second case, when the shortening velocity is lower, the stimulation time is longer, so the muscle tissue begins to generate force. However, even in this case, the stimulation time is still too short, and the force does not reach its maximum value.

On the other hand, dual issues arise when the shortening is too big, again for both high [Fig. 2(c)] and low [Fig. 2(d)] shortening velocity. In the first case, the force generated by the tissue is far from its maximum value, while, in the second case, the tissue has a sufficient time to generate its maximum force but, due to excessive contraction strain, it is not able to keep it constant for the entire stimulation period.

Our results pointed out that X-METs are able to produce power and maintain it during the entire isovelocity shortening period only when the shortening is in the range of $5 \% \mathrm{~L}_{0}$ and $12 \% \mathrm{~L}_{0}$, and the shortening velocity is in the range of $0.25 \mathrm{~L}_{0} / \mathrm{s}$ and $1 \mathrm{~L}_{0} / \mathrm{s}$. Given that the X-MET's average length is approximately $20 \mathrm{~mm}$ the corresponding values of velocity are in the range of 5 and $20 \mathrm{~mm} / \mathrm{s}$. According to these ranges the electrical stimulation, and therefore the shortening, must have a duration between 100 and $600 \mathrm{~ms}$ to yield a proper development of the power. Fig. 3 shows an example

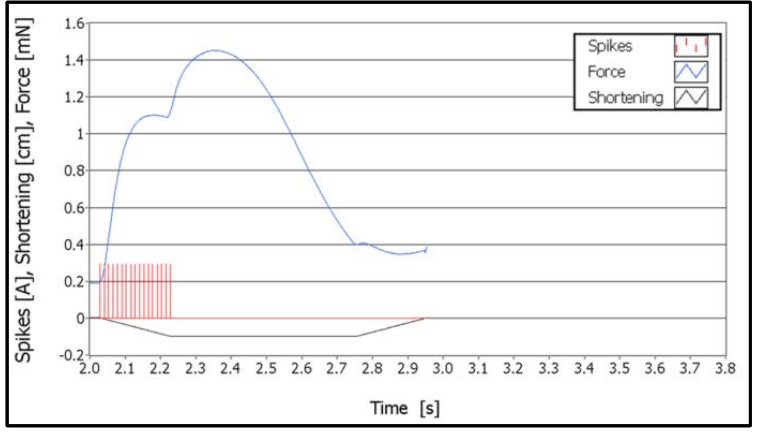

Fig. 3. Force development (blue) during an isovelocity test performed with optimal testing parameters. Red line represents the electrical pulses and black line shows the shortening.

of isovelocity contraction where the amount of shortening and shortening velocity is in the optimal range, as previously determined. The force development is in agreement with that of EDL and Soleus muscles [25], [26]: the force increases up to the maximum value and is maintained for the entire stimulation time. Once the shortening ends, the tissue is held in isometric conditions and the force increases.

\section{B. Measurement of mechanical power}

Once optimal ranges of shortening and shortening velocity were determined, we measured the mechanical power of $11 \mathrm{X}$-METs by subjecting them at 16 different isovelocity tests. The shortening varied between $5 \% \mathrm{~L}_{0}$ and $12 \% \mathrm{~L}_{0}$, and the shortening velocity varied between $0.25 \mathrm{~L}_{0} / \mathrm{s}$ and $1 \mathrm{~L}_{0} / \mathrm{s}$. 


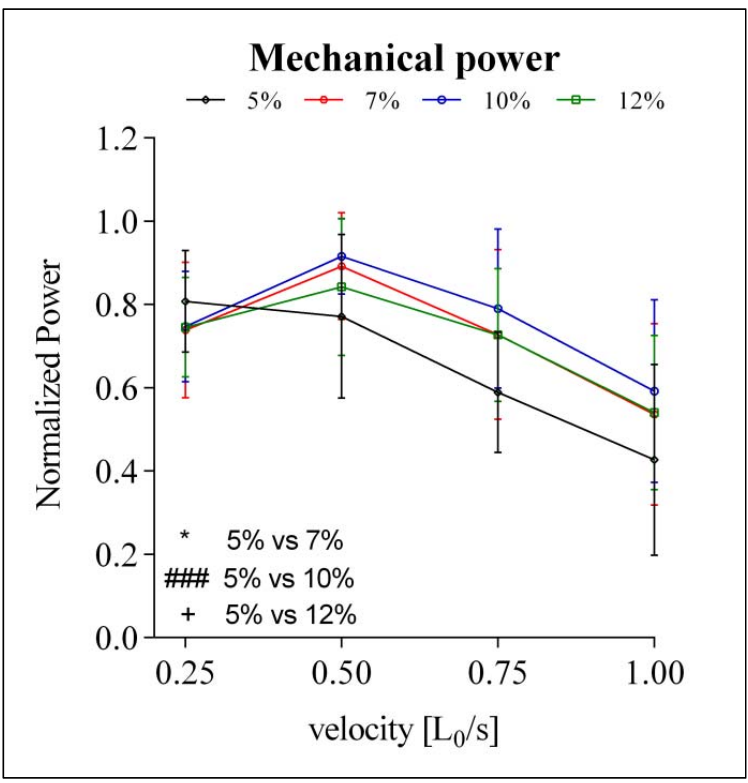

Fig. 4. Mean $\pm \mathrm{SD}$ of the mechanical power normalized by the maximum power measured when the shortening and the shortening velocity were in the optimal ranges of stimulation.* $5 \%$ versus 7\% $p<0.05$; \#\# 5\% versus $10 \% * p<0.001 ;+5 \%$ versus $12 \% p<0.05$.

The optimal length of the tested samples was $20.1 \pm 2.9 \mathrm{~mm}$. For each test, the power was obtained multiplying the constant speed by the maximum force during each stimulation. Fig. 4 shows the summary of all the tests.

The two way ANOVA revealed that both the factors, shortening $(p<0.01)$ and shortening velocity $(p<0.0001)$, significantly affected the power generated by the tissue. Results showed also that the power developed when shortening the tissue at $5 \% \mathrm{~L}_{0}$ was lower than when shortening the tissue at the other tested conditions, being this difference significant in comparison to $7 \% \mathrm{~L}_{0}(p<0.05), 10 \% \mathrm{~L}_{0}(p<0.001)$, and $12 \% \mathrm{~L}_{0}(p<0.05)$ of shortening. On the other hand, for all the shortenings between $7 \% \mathrm{~L}_{0}$ and $12 \% \mathrm{~L}_{0}$ the maximum power generated by X-METs was obtained when the tissue was shortened at the constant speed of $0.5 \mathrm{~L}_{0} / \mathrm{s}$. Posthoc tests revealed that the power generated for $v=0.5 \mathrm{~L}_{0} / \mathrm{s}$ was significantly higher than that generated for $0.25 \mathrm{~L}_{0} / \mathrm{s}$ $(p<0.01), 0.75 \mathrm{~L}_{0} / \mathrm{s}(p<0.01)$, and $1 \mathrm{~L}_{0} / \mathrm{s}(p<0.0001)$ when the shortening was equal to $7 \% \mathrm{~L}_{0}$, than that generated at $0.25 \mathrm{~L}_{0} / \mathrm{s}(p<0.01), 0.75 \mathrm{~L}_{0} / \mathrm{s}(p<0.05)$, and $1 \mathrm{~L}_{0} / \mathrm{s}$ $(p<0.0001)$ when the shortening was equal to $10 \% \mathrm{~L}_{0}$, than that generated at $0.75 \mathrm{~L}_{0} / \mathrm{s}(p<0.05)$ and $1 \mathrm{~L}_{0} / \mathrm{s}(p<0.001)$ when the shortening was equal to $12 \% \mathrm{~L}_{0}$. In addition, the power measured when the specimens were shortened at $7 \%$ $\mathrm{L}_{0}$ and $10 \% \mathrm{~L}_{0}$ was higher than when tested at the two other conditions, being also significant in comparison to of $5 \% \mathrm{~L}_{0}$.

The maximum power we measured with our testing protocol was found to be $2.06 \pm 0.80 \mu \mathrm{W}$ on average which, normalized by the specimens' weight, corresponds to $1.03 \pm 0.4 \mathrm{~W} / \mathrm{kg}$.

\section{Hill's Model of the Force Velocity Relationship}

Fig. 5 shows an example of the hyperbolic model proposed by Hill, superimposed to the experimental points, in the case

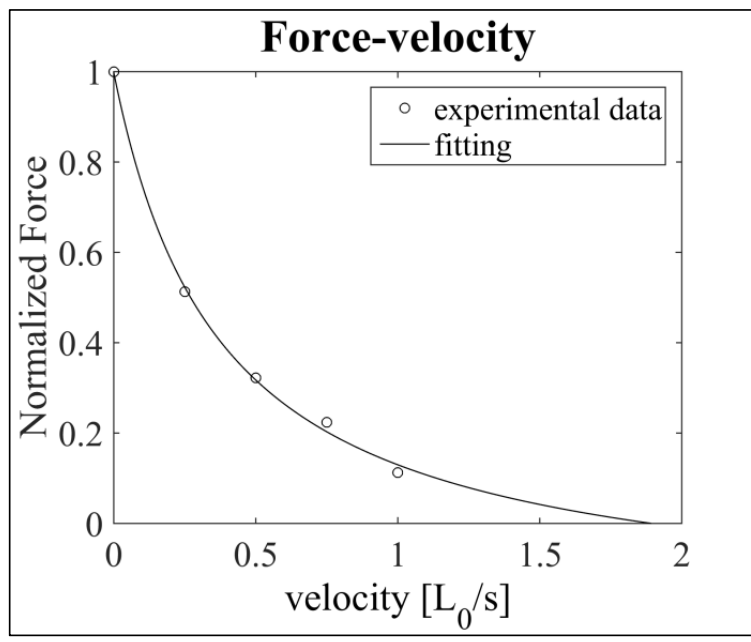

Fig. 5. Hyperbolic model proposed by Hill superimposed to the experimental points normalized to the maximum isometric force (F/Fmax) in the case of shortenings of $10 \% \mathrm{~L}_{0}\left(R^{2}=0.9982\right)$.

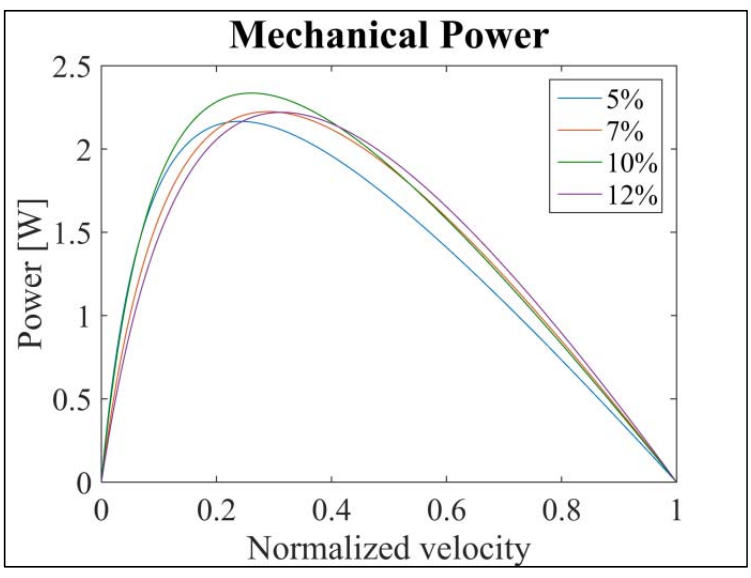

Fig. 6. Mechanical power calculated by the mathematical model for all the tested conditions. The optimal velocity of shortening is highlighted for the $10 \% \mathrm{~L}_{0}$ condition. Normalized velocity is $v / v_{\max }$.

of $10 \% \mathrm{~L}_{0}$ of shortening. To allow for a proper comparison among all the specimens, the force was normalized by the maximum force each specimen generated in isometric conditions (measured in the second part of the experimental protocol), and the shortening velocity was normalized by $\mathrm{L}_{0}$. It is worth noting how Hill's equation fit very well to our experimental data, with $R^{2}$ values always higher than 0.994 .

Once the mathematical model was obtained, we proceeded calculating the maximum shortening velocity, an important parameter used to evaluate skeletal muscle contractile properties. Of note, the maximum shortening velocity can be obtained only through a mathematical extrapolation, since it corresponds to the velocity the tissue would have when shortening against a null load [32], [33]. The average maximum shortening velocity was measured to be $1.84 \pm 0.57 \mathrm{~L}_{0} / \mathrm{s}$ on average, a value in high agreement with that proposed in the literature for red fiber bundles [27].

Furthermore, by multiplying the values of force predicted through equation 1 by the shortening velocity between zero 
TABLE I

Summary of all the Data Obtained From Experimental Tests and Mathematical Model Data are Mean \pm SD

\begin{tabular}{|c|c|c|c|c|}
\hline $\begin{array}{c}\mathrm{W}_{\max } \text { measured } \\
{[\mu \mathrm{W}]}\end{array}$ & $\begin{array}{c}\mathrm{W}_{\max } \text { estimated } \\
{[\mu \mathrm{W}]}\end{array}$ & $\begin{array}{c}\mathrm{V}_{\max } \\
{\left[\mathrm{L}_{0} / \mathrm{s}\right]}\end{array}$ & $\begin{array}{c}\mathrm{v}_{\text {opt }} \\
{\left[\mathrm{v} / \mathrm{v}_{\max }\right]}\end{array}$ & $\begin{array}{c}\mathrm{F}_{\text {opt }} \\
{\left[\mathrm{F} / \mathrm{F}_{\max }\right]}\end{array}$ \\
\hline $2.06 \pm 0.80$ & $2.08 \pm 0.78$ & $1.84 \pm 0.57$ & $0.27 \pm 0.03$ & $0.26 \pm 0.03$ \\
\hline
\end{tabular}

and the maximum value just estimated, it was possible to determine the power over the entire range of shortening velocity, in order to compute the maximum power, the optimal shortening velocity and optimal force, as shown in Fig. 6.

The maximum power obtained through the use of the mathematical model was $2.08 \pm 0.78 \mu \mathrm{W}$, on average, that corresponds to a normalized power of $1.04 \pm 0.38 \mathrm{~W} / \mathrm{kg}$, on average. This value of maximum power has been achieved when the velocity was around $27 \%$ of the maximum shortening velocity, and the force was around $26 \%$ of the maximum isometric force. Interestingly, these values are very close to that previously determined for Soleus muscles [24], $v_{\text {opt }}=27 \%$ $v_{\max }$ and $F_{\mathrm{opt}}=22 \% F_{\max }$. The shortening velocity was also very close to that obtained for bundles of red fibers of dogfish, $v_{\text {opt }}=29 \% v_{\max }$, while in this case the optimal force was slightly different, $F_{\text {opt }}=36 \% F_{\max }$ [25]. At this point, it has to be noted that maximum shortening velocity has been demonstrated to be the same if measured through the isovelocity shortening technique and through the isotonic shortening technique [34], while the maximum power measured through the isovelocity technique resulted lower than that measured with the isotonic technique. The difference relies in the fact that the power measured through the isotonic technique is computed as the product of a constant force by the peak of shortening velocity, namely a power maintained only for a very short time, representing the part of the power due to the activation of the muscle's fast fibers. In the isovelocity technique, the power is computed multiplying the constant velocity by the maximum force generated during the shortening that, on the contrary, is maintained for a longer time. The power measured through this technique is, therefore, more representative of the power generated by muscles mainly composed of slow twitch fibers, as the ex vivo muscle engineered tissue tested in this paper.

Table I displays the maximum power measured with the experimental tests and the mathematical model, the maximum shortening velocity and the optimal shortening velocity and optimal force.

It is interesting to note that the maximum power values measured with the isovelocity technique and through the modeling to the Hill's equation are almost identical. This is because the optimal shortening velocity calculated with the mathematical model is around $27 \%$ of the maximum shortening velocity, which is around $0.497 \mathrm{~L}_{0} / \mathrm{s}$, really close to one of the four points we chose to perform the isovelocity shortening, $v=$ $0.5 \mathrm{~L}_{0} / \mathrm{s}$. The maximum power we measured for our model of ex vivo muscle engineered tissues is about 40 times lower than that previously proposed in the literature for red fibers bundles, $30 \mathrm{~W} / \mathrm{kg}$ [27], and Soleus muscle $50 \mathrm{~W} / \mathrm{kg}$ [26], [25]. Even if this difference is still high, it has to be noted that the maximum specific forces previously measured for different $e x$ vivo muscle engineered tissues are more than two order of magnitude lower than skeletal muscles, pointing out that the $\mathrm{X}$-MET has a kinetic behavior close to that of native muscles. This is also confirmed by the fact that the maximum shortening velocity we measured is in high agreement with that proposed in the literature for red bundles of fibers and for slow-twitch Soleus muscles [27], [32], [35], [36].

On the other hand, such a big difference in the values of generated power is probably due to the lack of innervations that distinguishes ex vivo muscle tissues from real muscles. It has been demonstrated that tendons amplify the mechanical power developed by a muscle [37], [38], so that the absence of tendons in ex vivo engineered tissues could result in lower values of generated power. In this context, the capability of measuring the power developed by ex vivo engineered muscle, as proposed in this paper, is a fundamental tool to improve the generation techniques of these tissues. Any amelioration in the cultivation procedure employed to generate ex vivo tissues may be quantified with our measurement protocol, with the final aim of generating tissues as close as possible to native muscles.

\section{CONCLUSION}

In this paper, we proposed an accurate measurement of the isotonic contractile properties of ex-vivo muscle tissues, which are crucial to obtain proper compatibility when transplanting the tissue back in the host body. To reach this aim we applied the isovelocity shortening contraction methodology, a technique already employed to measure fast and slow skeletal muscle power, to the X-MET, an ex vivo muscle engineered tissue, and determined the optimal testing conditions that let this tissue generate and maintain power similar to that of native muscles. The experimental results have been fit to the Hill's model of force-velocity relationship to capture the maximum shortening velocity, the maximum power, and the shortening velocity and the force at which the tissue generated its maximum power. Results showed that most of these properties were consistent with that proposed in the literature for slowtwitch muscles, confirming that the measurement of isotonic properties is crucial to help improving the development of ex vivo muscle engineered tissues. In particular, the maximum power generated by the tested X-METs was of about $1 \mathrm{~W} / \mathrm{kg}$, and the maximum shortening velocity was predicted to be of about $1.8 \mathrm{~L}_{0} / \mathrm{s}$. Of note, a comprehensive measurement of the contribution that this tissue might bring to the power development during locomotion can be obtained only through the use of in vivo techniques. The next goal of our research will be therefore to measure the power developed by the entire mouse hind limb once the X-MET has been transplanted 
back to replace damaged tissue. Only a right activation of the transplanted tissue together with the host ones will bring the surgical limb to generate as power as a healthy control.

\section{REFERENCES}

[1] I. Martin, R. Quarto, B. Dozin, and R. Cancedda, "Producing prefabricated tissues and organs via tissue engineering," IEEE Eng. Med. Biol. Mag., vol. 16, no. 2, pp. 73-80, Mar./Apr. 1997.

[2] H. Hagenmüller, M. Hitz, H. P. Merkle, L. Meinel, and R. Müller, "Design and validation of a novel bioreactor principle to combine online micro-computed tomography monitoring and mechanical loading in bone tissue engineering," Rev. Sci. Instrum., vol. 81, no. 1, 2010, Art. no. 014303.

[3] C. A. Rossi, M. Pozzobon, and P. De Coppi, "Advances in musculoskeletal tissue engineering: Moving towards therapy," Organogenesis, vol. 6, no. 3, pp. 167-172, 2010.

[4] A. D. Bach et al., "A new approach to tissue engineering of vascularized skeletal muscle," J. Cell. Mol. Med., vol. 10, no. 3, pp. 716-726, 2006.

[5] A. Godier-Furnemont and G. Vunjak-Novakovic, "Cardiac muscle tissue engineering," in Biomaterials Science: An Introduction to Materials, 3rd ed. Amsterdam, The Netherlands: Elsevier, 2013, pp. 1262-1276. doi: 10.1016/B978-0-08-087780-8.00117-0.

[6] M. V. Chor and W. Li, "A permeability measurement system for tissue engineering scaffolds," Meas. Sci. Technol., vol. 18, no. 1, pp. 208-216, 2007. doi: 10.1088/0957-0233/18/1/026.

[7] A. Schiavi, C. Guglielmone, F. Pennella, and U. Morbiducci, "Measurement science and technology acoustic method for permeability measurement of tissue-engineering scaffold," Meas. Sci. Technol., vol. 23, no. 10, 2012, Art. no. 105702.

[8] C. G. Simon, J. S. Stephens, S. M. Dorsey, and M. L. Becker, "Fabrication of combinatorial polymer scaffold libraries," Rev. Sci. Instrum., vol. 78, no. 7, 2007, Art. no. 072207.

[9] X. Li et al., "Preparation and osteogenic properties of magnesium calcium phosphate biocement scaffolds for bone regeneration," J. Instrum., vol. 8, no. 7, 2013, Art. no. C07010

[10] A. K. Saxena, J. Marler, M. Benvenuto, G. H. Willital, and J. P. Vacanti, "Skeletal muscle tissue engineering using isolated myoblasts on synthetic biodegradable polymers: Preliminary studies," Tissue Eng., vol. 5, no. 6, Jan. 2007. doi: 10.1089/ten.1999.5.525.

[11] M. K. Smith, K. W. Riddle, and D. J. Mooney, "Delivery of hepatotrophic factors fails to enhance longer-term survival of subcutaneously transplanted hepatocytes," Tissue Eng., vol. 12, no. 2, pp. 235-244, 2006.

[12] K. J. L. Burg and T. Boland, "Minimally invasive tissue engineering composites and cell printing," IEEE Eng. Med. Biol. Mag., vol. 22, no. 5, pp. 84-91, Sep./Oct. 2003.

[13] W. Bian and N. Bursac, "Cellular/tissue engineering," IEEE Eng. Med. Biol. Mag., vol. 27, no. 5, pp. 109-113, Sep./Oct. 2008.

[14] C. A. Powell, B. L. Smiley, J. Mills, and H. H. Vandenburgh, "Mechanical stimulation improves tissue-engineered human skeletal muscle," Amer. J. Physiol. Cell Physiol., vol. 283, no. 5, pp. C1557-C1565, 2002.

[15] M. Kageshima, T. Maruyama, T. Akama, and T. Nakamura, "Novel magnetic indenter for rheological analysis of thin biological sheet for regenerative medicine," Rev. Sci. Instrum., vol. 87, no. 7, 2016, Art. no. 074302 .

[16] M. W. Laschke and M. D. Menger, "Life is 3D: Boosting spheroid function for tissue engineering," Trends Biotechnol., vol. 35, no. 2, pp. 133-144, Feb. 2017.

[17] R. Leitão et al., "Study of response to zinc in human prostate spheroids using X-ray microfluorescence," IEEE Trans. Instrum. Meas., vol. 64, no. 8, pp. 2121-2126, Aug. 2015.

[18] R. C. Strohman, E. Bayne, D. Spector, T. Obinata, J. Micou-Eastwood, and A. Maniotis, "Myogenesis and histogenesis of skeletal muscle on flexible membranes in vitro," Cell Develop. Biol., vol. 26, no. 2, pp. 201-208, Feb. 1990.

[19] R. G. Dennis, P. E. Kosnik, M. E. Gilbert, and J. A. Faulkner, "Excitability and contractility of skeletal muscle engineered from primary cultures and cell lines," Amer. J. Physiol. Cell Physiol., vol. 280, no. 2, pp. C288-C295, 2001.

[20] S. Carosio, L. Barberi, E. Rizzuto, C. Nicoletti, Z. Del Prete, and A. Musarò, "Generation of ex vivo-vascularized muscle engineered tissue (X-MET)," Sci. Rep., vol. 3, Mar. 2013, Art. no. 1420.

[21] E. Rizzuto, S. Carosio, M. Faraldi, S. Pisu, A. Musarò, and Z. Del Prete, "A DIC based technique to measure the contraction of a skeletal muscle engineered tissue," Appl. Bionics Biomech., vol. 2016, Feb. 2016, Art. no. 7465095.

[22] M. Caruel and L. Truskinovsky, "Physics of muscle contraction," Rep. Prog. Phys., Feb. 2018. doi: 10.1088/1361-6633/aa7b9e.
[23] E. Zemková, A. Cepková, M. Uvaček, and D. Hamar, "A new method to assess the power performance during a lifting task in young adults," Measurement, vol. 91, pp. 460-467, Sep. 2016.

[24] E. Zemková, P. Miklovič, A. Dunajčík, and D. Hamar, "Power as a parameter in the functional assessment of knee flexions and knee extensions on weight stack machines," Measurement, vol. 61, pp. 142-149, Feb. 2015.

[25] S. V. Brooks, J. A. Faulkner, and D. A. McCubbrey, "Power outputs of slow and fast skeletal muscles of mice.," J. Appl. Physiol., vol. 68, no. 3, pp. 1282-1285, 1990.

[26] G. S. Lynch, R. T. Hinkle, and J. A. Faulkner, "Power output of fast and slow skeletal muscles of MDX (dystrophic) and control mice after clenbuterol treatment," Exp. Physiol., vol. 85, no. 3, pp. 295-299, 2000.

[27] F. Lou, N. A. Curtin, and R. C. Woledge, "Isometric and isovelocity contractile performance of red muscle fibres from the dogfish Scyliorhinus canicula," J. Exp. Biol., vol. 205, no. 11, pp. 1585-1595, 2002.

[28] A. V. Hill, "The heat of shortening and the dynamic constants of muscle," Proc. Roy. Soc. B, Biol. Sci., vol. 126, no. 843, pp. 136-195, 1938.

[29] S. Pisu, L. Apa, M. Cosentino, A. Musarò, E. Rizzuto, and Z. Del prete, "Measuring the X-MET's maximum power: A preliminary study," in Proc. IEEE Int. Symp. Med. Meas. Appl. (MeMeA), Jun. 2018, pp. 1-5.

[30] S. Watanabe, S. Fukuhara, T. Fujinaga, and H. Oka, "Estimating the minimum stimulation frequency necessary to evoke tetanic progression based on muscle twitch parameters," Physiol. Meas., vol. 38, no. 3, p. 466, 2017.

[31] A. B. Schultz, J. A. Faulkner, V. A. Kadhiresan, and V. A. Kad, "A simple Hill element-nonlinear spring model of muscle contraction biomechanics," J. Appl. Physiol., vol. 70, no. 2, pp. 803-812, 2017. doi: 10.1152/jappl.1991.70.2.803.

[32] G. S. Lynch, R. T. Hinkle, J. S. Chamberlain, S. V. Brooks, and J. A. Faulkner, "Force and power output of fast and slow skeletal muscles from mdx mice 6-28 months old," J. Physiol., vol. 535, no. 2, pp. 591-600, 2001.

[33] S. V. Brooks and J. A. Faulkner, "Contractile properties of skeletal muscles from young, adult and aged mice," J. Physiol., vol. 404, no. 1, pp. 71-82, 1988.

[34] F. J. Julian, L. C. Rome, D. G. Stephensont, and S. Striz, "The maximum speed of shortening in living and skinned frog muscle fibres," J. Phisiol., vol. 370, no. 1, pp. 181-199, 1986.

[35] C. J. Barclay, "Mechanical efficiency and fatigue of fast and slow muscles of the mouse," J. Physiol., vol. 497, no. 3, pp. 781-794, 1996.

[36] C. J. Barclay, J. K. Constable, and C. L. Gibbs, "Energetics of fastand slow-twitch muscles of the mouse," J. Physiol., vol. 472, no. 1, pp. 61-80, 1993.

[37] Q. Zhang, X. Wang, M. Tian, X. Shen, and Q. Wu, "Modeling of novel compound tendon-sheath artificial muscle inspired by hill muscle model," IEEE Trans. Ind. Electron., vol. 65, no. 8, pp. 6372-6381, Aug. 2017.

[38] G. S. Sawicki, P. Sheppard, and T. J. Roberts, "Power amplification in an isolated muscle-tendon unit is load dependent," J. Exp. Biol., vol. 218, no. 22 , pp. 3700-3709, 2015.

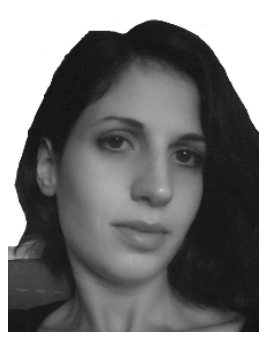

Simona Pisu received the master's degree in biomedical engineering and the Ph.D. degree in morphogenesis tissue engineering from the University of Rome La Sapienza, Rome, Italy, in 2013 and 2017, respectively.

She was involved in studying functional and biomechanical properties and neuromuscular junction functionality of muscles both isolated and in situ from control and pathological animal model, experimental protocols and in vitro measurement techniques for the determination and conditioning of biomechanical properties of 3-D engineered in vitro muscle tissues until 2018.

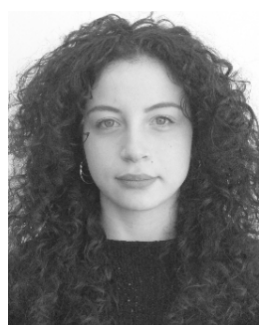

Marianna Cosentino received the B.S. degree in sanitary biotechnologies from the Catholic University of Rome, Rome, Italy, in 2015, and the M.S. degree in pharmaceutical biotechnologies from the University of Rome La Sapienza, Rome, in 2017, where she is currently pursuing the Ph.D. degree in morphogenesis and tissue engineering with the School of Biology and Molecular Medicine.

Her current research interests include the development and characterization of engineered muscle tissues and the study of epigenetic and biomedical signals. 


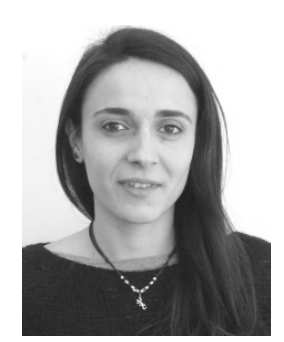

Ludovica Apa received the B.E. degree in clinical engineering and the M.E. degree in biomedical engineering from the University of Rome La Sapienza, Rome, Italy, in 2013, and 2016, respectively, where she is currently pursuing the Ph.D. degree in industrial and management engineering.

Her current research interests include tissue biomechanics, cell mechanotransduction, and digital image correlation techniques.

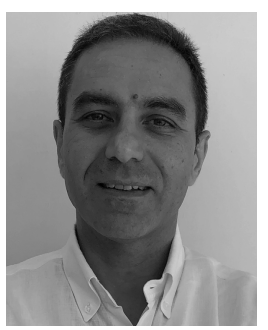

Antonio Musarò received the Ph.D. degree in biotechnology from the University of Rome La Sapienza, Rome, Italy.

He was a Research fellow in medicine with Harvard University, Boston, MA, USA. He was an Adjunct Associate Professor with Edith Cowan University, Joondalup, WA, Australia. He was a Chief with the Interuniversity Institute of Myology. He is currently a Full Professor in histology, embryology and biotechnology, with the University of Rome La Sapienza, where he is the Scientific Coordinator of the Ph.D. Program in morphogenesis and tissue engineering and the Director of Master in stem cells and genome editing. His current research interests include the molecular aspects of muscle homeostasis, regeneration, and wasting.

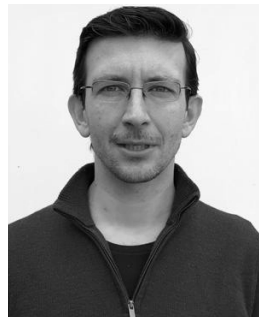

Emanuele Rizzuto received the master's degree in mechanical engineering and the Ph.D. degree in Cell Science and Morphogenesis from the University of Rome La Sapienza, Rome, Italy, in 2003 and 2008, respectively.

$\mathrm{He}$ is currently an Assistant Professor of industrial and biomedical measurements with the University of Rome La Sapienza. His current research spreads from the measurements for biomedical applications, such as the measurement of biological tissues' mechanical properties, and their involvement in specific pathologies, to the measurements for industrial applications, such as the measurement of lithium ion cell performances.

Dr. Rizzuto is a member of the Interuniversity Institute of Myology and the IEEE-IMS Instrument and Measurement Society.

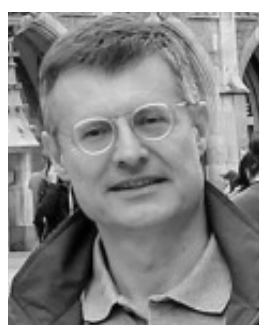

Zaccaria Del Prete is currently a Professor of industrial and biomedical measurements with the Department of Mechanical and Aerospace Engineering, University of Rome La Sapienza, Rome, Italy. He was a Visiting Scientist with the University of Massachusetts, Amherst, MA, USA. He is the Director of the Clinical and Biomedical Engineering Program, University of Rome La Sapienza. He has co-authored more than 75 international journals and conference articles, and two book chapters. He holds two patents. His current research interests include the spread from in vitro analysis of transgenic muscle fiber bio-mechanical properties to experimental analysis of mechanoreceptor neurons coding mechanism and of the mechanical properties of viscoelastic biological soft tissue and to static and dynamic characterization of fuel cell systems in the industrial field.

Dr. Del Prete has been a member of SEM Society for Experimental Mechanics, the IEEE-EMB Engineering in Medicine and Biology Society, and is currently a member of the IEEE-IMS Instrument and Measurement Society. 